\title{
CORRESPONDENCE
}

\section{Time to end US chimp studies}

As a physician, medical educator and former animal researcher, I agree that the US national discussion of chimpanzee experimentation should go beyond simple husbandry issues (Nature 474, $252 ; 2011)$. For ethical and scientific reasons, it is time for the United States to join other developed countries in ending invasive experiments on chimpanzees.

We now know a great deal about the awareness, intelligence and emotional responses of chimpanzees. Scientists from my organization, for example, have found that invasive experiments on chimpanzees can induce symptoms of depression, anxiety and compulsive behaviours that are similar to mood and anxiety disorders seen in traumatized humans (H. R. Ferdowsian et al. PLoS ONE 6, e19855; 2011).

Although chimps are humankind's closest genetic relatives, we show significant differences in our gene expression, physiology and disease susceptibility. It is becoming increasingly evident that chimpanzee experiments are not improving our understanding and treatment of human disease. Billions of dollars and decades of research using chimpanzees have not produced effective vaccines for hepatitis C, HIV, malaria or other diseases, nor have they provided insight into cancer, neurological diseases or psychiatric disorders. However, the process has inflicted extensive and often lifelong pain and suffering on these animals.

John J. Pippin Physicians Committee for Responsible Medicine, Washington DC, USA. jpippin@pcrm.org

\section{E. coli outbreak exposed tech gaps}

As well as the organizational mismanagement of the recent Escherichia coli outbreak in Germany (Nature 474, 251; 2011), the technical underdevelopment of the country's medical microbiology institutes is staggering, given that Germany is the largest economy in Europe. Such shortcomings leave the country unprotected against attacks by highly virulent agents of natural or bioterrorist origin.

For example, none of these institutes is set up for rapid sequencing or mass screening of major pathogenic agents using the polymerase chain reaction (PCR). Most of the labs still rely solely on Robert Koch's lengthy culture methods, even though analysis of a known pathogen could be reduced to a few hours by using culture enrichment combined with high-throughput real-time PCR. Such an analysis during the recent outbreak would have increased the number of samples tested and probably saved lives.

Rainer Fislage St Wendel, Germany. rainer.fislage@biophenium.de

\section{Dam not sole cause of Chinese drought}

China's Yangtze River is suffering its worst drought for more than 50 years (Nature doi:10.1038/ news.2011.315; 2011). Although people blame the Three Gorges Dam for making matters worse, other factors have also contributed.

In response to April and May's severe water shortage, central government ordered the release of 50 billion cubic metres of water from reservoirs in the Yangtze basin, most of which came from the Three Gorges Reservoir, with some from other reservoirs used for power generation. The result is a cumulative toll on the river's large-scale hydrological balance.

Extensive land reclamation in the middle and lower reaches of the river has exacerbated the drought by removing or shrinking many natural lakes across the river basin. Worse, more than $80 \%$ of the remaining lakes are no longer connected with the river, seriously limiting their capacity for buffering the water supply.

Excessive pumping of groundwater is a significant contributor to the current drought (see go.nature.com/ b6Srkg; in Chinese), as are channel incisions caused by loss of sediment and sand mining.

The drought's severity threatens China's south-north water-diversion project, a huge trans-basin scheme to ease the water shortage in northern China. X. X. Lu, Xiankun Yang, Siyue Li National University of Singapore, Singapore. geoluxx@nus.edu.sg

\section{Green labelling being misused}

'Green' labelling of items produced sustainably has become a much-publicized tool of the environmental movement. But green-label criteria that were developed for forestry are now being inappropriately applied to agricultural crops - with unacceptable risks to wildlife.

The high conservation value (HCV) concept was originally intended for timber harvested without harming large forest blocks or critically endangered flora and fauna. It has since been extended to some of the most rapidly expanding crops in the tropics - namely, oil palm, soya bean, sugar cane and cacao — ostensibly to ensure their 'sustainable' production.

The combined area of these four crops increased by 36.5 million hectares between 1999 and 2008, largely in countries of exceptional biodiversity, such as Brazil and Indonesia, and largely at the expense of forest. The hope is that green labelling through $\mathrm{HCV}$ will stem this tide of habitat destruction and biodiversity loss.

But many of the world's most intact and biodiverse tropical forests (including the Amazon Basin, Congo and New Guinea) harbour few critically endangered species. In such cases, the only HCV criterion that is likely to prevent forest conversion to agriculture is the one protecting large expanses of habitat.

Unfortunately, the round tables for oil palm, soya bean, sugar cane and cacao have decided that only forest blocks larger than 20,000-500,000 hectares (depending on the country) are eligible for protection under this criterion. So crops replacing areas of forest below these thresholds can perversely carry a green label, even though these thresholds are dangerously high for wildlife survival.

The size of HCV thresholds must be drastically reduced so that green-labelled crops are not simply 'greenwash'.

David P. Edwards, Brendan Fisher, David S. Wilcove Princeton University, New Jersey, USA.

dpedward@princeton.edu

\section{CONTRIBUTIONS}

Correspondence may be sent to correspondence@ nature.com after consulting the author guidelines at go.nature.com/cmchno. Readers are also welcome to comment online on anything published in Nature: www.nature.com/nature. 